\title{
Analytical models of contact interaction of casing centralizers with well wall
}

\author{
I. Shatskyi ${ }^{\mathrm{a}}$, A. Velychkovych ${ }^{\mathrm{b}^{*}}$, I. Vytvytskyi ${ }^{\mathrm{b}}$ and M. Seniushkovych ${ }^{\mathrm{b}}$
}

${ }^{a}$ Ivano-Frankivsk Branch of Pidstryhach-Institute for Applied Problems in Mechanics and Mathematics, NAS of Ukraine, Department of modelling of damping systems, Ukraine

${ }^{b}$ Ivano-Frankivsk National Technical University of Oil and Gas, Ukraine

\begin{tabular}{l}
\hline A R T I C L EI N F O \\
\hline Article history: \\
Received 25 December, 2018 \\
Accepted 17 June 2019 \\
Available online \\
17 June 2019 \\
\hline Keywords: \\
Casing centralizer \\
Rod \\
Contact interaction \\
Stressed state \\
Rigidity \\
Strength
\end{tabular}
\begin{abstract}
A B S T R A C T
The problem of interaction of rod centralizers of casing with a well wall is considered. The paper aims at studying the ways of fastening of centralizer's ends on the parameters of its rigidity and strength. Knowledge of these characteristics is necessary for the assessment of casing column drift and well completion quality. For simulation of the working links of the centralizers, the classical linear theory of smooth rods, rigid along the axis and elastic on bending is used. Stress-strain state of bow-type rod with six different variants of fastening under conditions of point contact is studied. The analytical relations between the contact force and the mutual approach of the column and the well wall, as well as the expressions for the admissible contact loads, are established. The way of fastening the rod significantly influences these characteristics; in particular, the fact of the presence or absence of freedom of mutual movement of its ends along the pipe is decisive. The engineering formulas, which serve as two-side estimations of the rigidity and strength of the real structures of centering devices, are obtained. A comparative analysis of mechanical properties of centralizers has been conducted.
\end{abstract}

(C) 2019 Growing Science Ltd. All rights reserved.

\section{Introduction}

Directional drilling of deep oil and gas wells is currently popular in the world, whereby the axis of wells is often a spatial curve. Providing the required gap between the well wall and the casing has a determining influence on the quality of the cementation of the production casing, since it reduces the probability of cross flows (Renpu, 2011; Larin et al., 2013). The more complex the configuration of the well axis, the more difficult it is to ensure the coaxial position of the casing when it is lowered into such a well. Centralizers are important links of the technical equipment of the casing. Placing a large number of centering devices on the casing can significantly impede the descent of the pipes into the hole and increase the overall cost of the well casing. On the other hand, too few centralizers on the casing string will not provide the proper quality of the cement job. In order to develop a method for determining the required number of centering devices and their location along the length of the casing string, first, it is necessary to study the mechanical properties of the centralizers, in particular the parameters of rigidity and strength at contact load. The above-mentioned encouraged study of the problems of contact interaction of the centralizer links with the well walls.

* Corresponding author.

E-mail addresses: ㅅvelychkovych@ukr.net (A. Velychkovych)

(C) 2019 Growing Science Ltd. All rights reserved.

doi: 10.5267/j.esm.2019.6.002 


\section{State of question and statement of research problem}

Different types of centering devices are used to center the casing in an open borehole. The most commonly used when drilling vertical and directional wells are typical (Fryz, 2003; Casing Accessories, 2016) elastic rod "bow" type centering devices (Fig. 1). The existing reference documents and the literary sources known to us (Liu \& Weber, 2012; Kozhevnikov et al., 2015; Kotskulych et al., 2016; Vytvytskyi et al., 2017) provide a number of recommendations for the installation of bow-spring centralizers, depending on the configuration of the well axis.

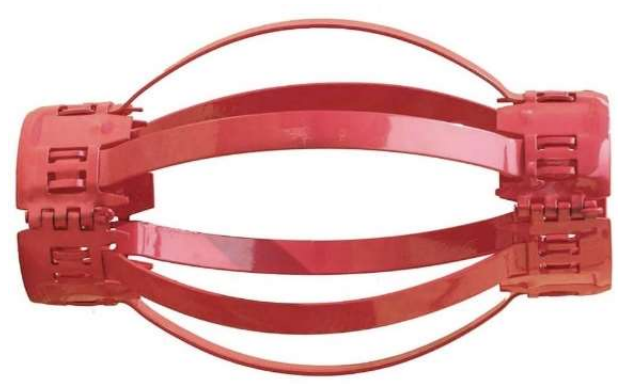

(a)

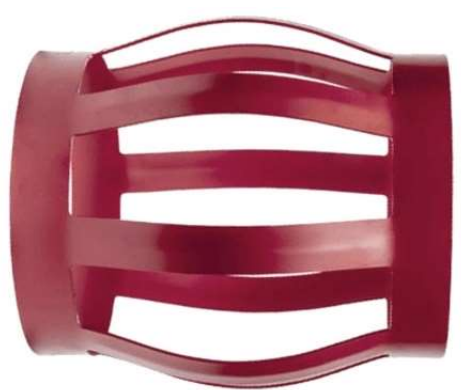

(b)

Fig. 1. Natural samples of centralizers with moveable $(a)$ and fixed $(b)$ fastening of elastic links.

General problems of interaction of columns with the borehole were considered, in particular, in the papers (Aleksandrov, 1982; Vlasiy et al., 2017; Levchuk, 2018; Kryzhanivskyi et al., 2018). Various approaches to the engineering simulation of contact phenomena in rod structures using classical theories of rods are proposed in publications (Mossakowskii et al., 1978; Bedzir et al., 1995; Neto et al., 2014; Shats'kyi et al., 2016; Popadyuk et al., 2016; Jawed et al., 2018). Some contact problems for thin shells and their systems are considered in classical statement (Shats'kyi, 2005; Shats'kyi \& Makoviichuk, 2005; Shats'kyi \& Makoviichuk, 2009; Shatskii \& Makoviichuk, 2011; Velichkovich \& Dalyak, 2015; Velichkovich et al., 2018). The numerical-analytical (Tikhonov et al., 2014) and experimental (Money et al., 2018) studies touch upon the analysis of the mechanical properties of the centralizers and the interaction of the column-equipped well with the well wall. The authors of the paper (Shatskyi et al., 2014) have initiated the procedure for formulation of analytical bilateral assessment of the rigidity and strength of the hinged centralizers of the casing.

The purpose of this paper is to study the influence of the entire spectrum of structural features of elastic cyclic symmetrical rod centralizers on mechanical characteristics of their rigidity and strength. There are six types of centralizer designs with various ways of fastening of rod links. The problems of contact interaction of the elastic links of the centralizer with the casing and the well wall are formulated and analytically solved.

\section{Main Content of the Paper}

\subsection{Formulation of the Contact Problems}

Let the casing pipe before tripping in is equipped with a centralizer, which consists of a cyclic system of bow-shaped rods mounted on the common rings enclosing the pipe. Let's consider the contact interaction of one link of centralizer with the well wall. The degree of pressing the column against the wall is considered such for which it is possible to look into the contacts of the central part of the centralizer with the well wall, and its ends with the pipe as point ones with concentrated contact reactions. The profile of the rod is bow-type, and its cross-section, for example, is rectangular $(b \times h)$. Let it be 
given the convergence of the pipe with the wall or clamping force $(P)$. We studied the influence of the conditions of fastening of the links (moveable scheme 1 and fixed scheme 2 (Fig. 2) for the hinged centralizer, moveable scheme 3 and fixed scheme 4 (Fig. 3) for the cast centralizer, and moveable scheme 5 and fixed scheme 6 (Fig. 4) for a welded centralizer) on a stress-strain state of the rod. To simulate the working link of the centralizer, we used the classical linear theory of curvilinear rods (Rekach, 2010; Gere \& Goodno, 2012). Let $f$ is the lift boom of centralizer link, $2 l$ - the length of the projection of the rod on the abscissa axis, and $2 \alpha-$ arc span. The rod is considered to be shallow. At the same time we neglect the values $O(f / l)^{2}$ and $O(h / f)^{2}$ compared to one. From the inequality $(f / l)^{2}<<1$, follows, that $1 / R \approx 2 f / l^{2}, \alpha \approx 2 f / l$. In turn, this means that for an inclined rod, the parabolic, circular or cycloid profiles are assumed to be identical and are described by an arc-like curve with a small constant curvature.
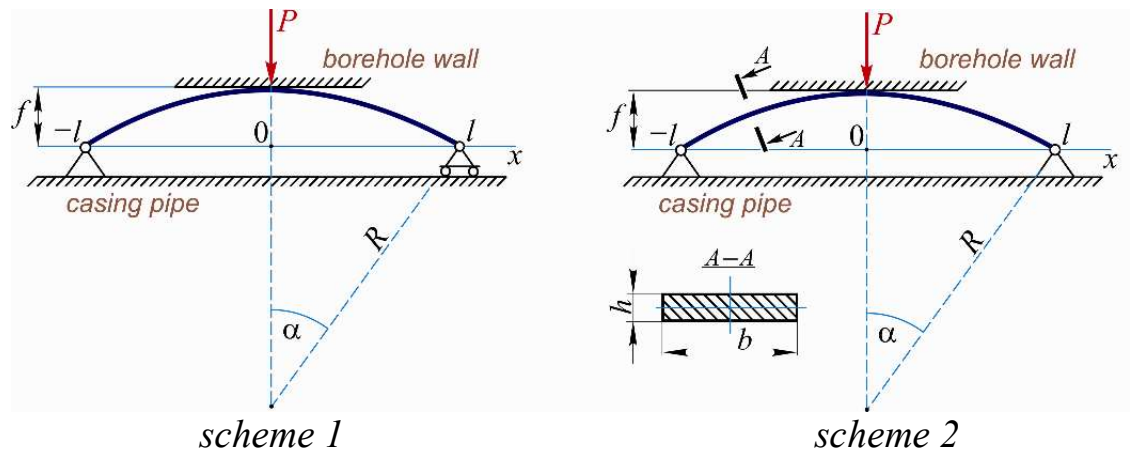

Fig. 2. Schemes of contact interaction of the hinged centralizer link with the well wall: scheme $1-$ axially moveable fastening, scheme 2 - axially fixed fastening.

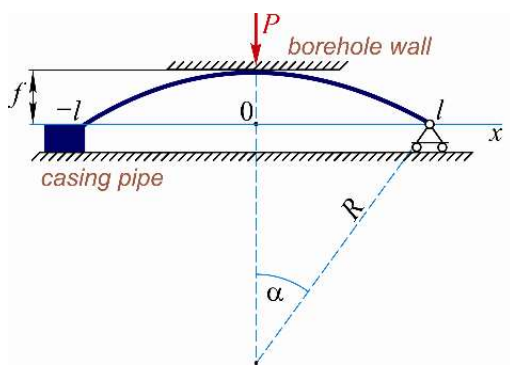

scheme 3

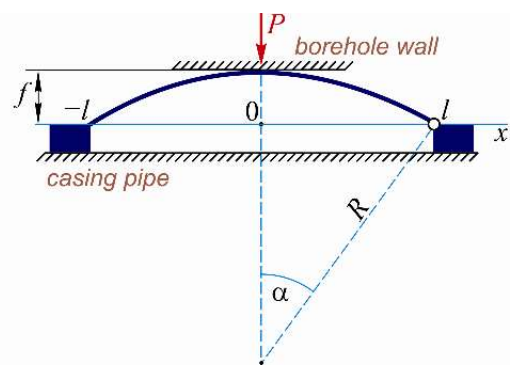

scheme 4

Fig. 3. Schemes of contact interaction of the cast centralizer link with the well wall: scheme 3 - axially moveable fastening, scheme 4 - axially fixed fastening.

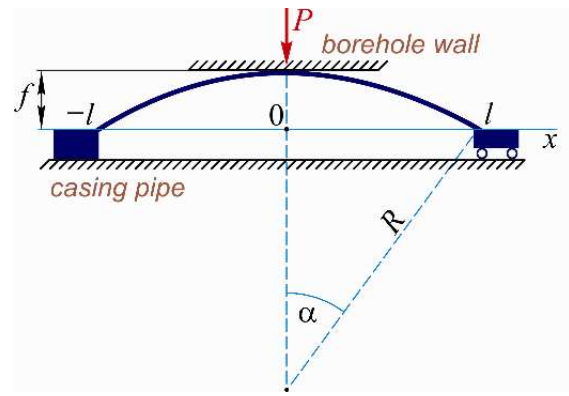

scheme 5

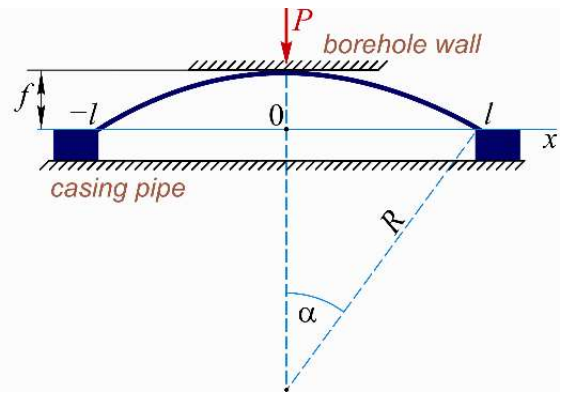

scheme 6

Fig. 4. Schemes of contact interaction of the welded centralizer link with the well wall: scheme 5 axially moveable fastening, scheme 6 - axially fixed fastening. 
Physical assumptions treat the rod as rigid along the curvilinear axis and elastic for bending. Let's write the key equations of the model:

equilibrium equation for an inclined rod

$$
\frac{d N}{d x}=0, \frac{d Q}{d x}+\frac{N}{R}=-P \delta(x), \frac{d M}{d x}-Q=0, \quad x \in(-l, l)
$$

constitutional relations

$$
\frac{d u}{d x}+\frac{w}{R}=0, \quad M=E J \frac{d^{2} w}{d x^{2}}, \quad x \in(-l, l) .
$$

here $N, Q$ are axial and transverse forces, $M$ is bending moment, $E J$ is rigidity of the rod on the bend, $u$ is tangential displacement, $w$ is rod deflection, $x$ is coordinate, $\delta(x)$ is Dirac function. Note that in the transition from the polar to the Cartesian coordinate system for small $\alpha$ for the components of the vectors of force and displacement there is a ratio:

$$
\begin{aligned}
& N_{x}( \pm l)=N( \pm l) \pm Q( \pm l) \alpha, N_{\mathrm{y}}( \pm l)=\mp N( \pm l) \alpha+Q( \pm l) \\
& u_{x}( \pm l)=u( \pm l) \pm w( \pm l) \alpha, u_{\mathrm{y}}( \pm l)=\mp u( \pm l) \alpha+w( \pm l) .
\end{aligned}
$$

Then the boundary conditions of fastening the ends of the rod $x= \pm l$ and its point $(x=0)$ interaction with a well wall are written as:

for scheme 1

$$
\begin{aligned}
& M(-l)=0, u_{x}(-l) \equiv u(-l)+w(-l) \alpha=0, u_{y}(-l) \equiv w(-l)+u(-l) \alpha=0 \\
& N_{x}(l) \equiv N(l)-Q(l) \alpha=0, M(l)=0, u_{y}(l) \equiv w(l)-u(l) \alpha=0, w(0)=-\Delta
\end{aligned}
$$

for scheme 2

$$
\begin{aligned}
& M(-l)=0, u_{x}(-l) \equiv u(-l)-w(-l) \alpha=0, u_{y}(-l) \equiv w(-l)+u(-l) \alpha=0, \\
& M(l)=0, u_{x}(l) \equiv u(l)+w(l) \alpha=0, u_{y}(l) \equiv w(l)-u(l) \alpha=0, w(0)=-\Delta
\end{aligned}
$$

for scheme 3

$$
\begin{aligned}
& u_{x}(-l) \equiv u(-l)-w(-l) \alpha=0, u_{y}(-l) \equiv w(-l)+u(-l) \alpha=0, \vartheta(-l)=0 \\
& N_{x}(l) \equiv N(l)-Q(l) \alpha=0, M(l)=0, u_{y}(l) \equiv w(l)-u(l) \alpha=0, w(0)=-\Delta
\end{aligned}
$$

for scheme 4

$$
\begin{aligned}
& u_{x}(-l) \equiv u(-l)-w(-l) \alpha=0, u_{y}(-l) \equiv w(-l)+u(-l) \alpha=0, \vartheta(-l)=0, \\
& M(l)=0, u_{x}(l) \equiv u(l)+w(l) \alpha=0, u_{y}(l) \equiv w(l)-u(l) \alpha=0, w(0)=-\Delta ;
\end{aligned}
$$

for scheme 5

$$
u_{x}(-l) \equiv u(-l)-w(-l) \alpha=0, u_{y}(-l) \equiv w(-l)+u(-l) \alpha=0, \vartheta(-l)=0,
$$




$$
N_{x}(l) \equiv N(l)-Q(l) \alpha=0, \vartheta(l)=0, u_{y}(l) \equiv w(l)-u(l) \alpha=0, w(0)=-\Delta
$$

for scheme 6

$$
\begin{aligned}
& u_{x}(-l) \equiv u(-l)-w(-l) \alpha=0, u_{y}(-l) \equiv w(-l)+u(-l) \alpha=0, \vartheta(-l)=0, \\
& u_{x}(l) \equiv u(l)+w(l) \alpha=0, u_{y}(l) \equiv w(l)-u(l) \alpha=0, \vartheta(l)=0, w(0)=-\Delta .
\end{aligned}
$$

\subsection{Analytical Solutions and their Analyze}

Applying the scheme of the method of initial parameters (Rekach, 2010), general solution of Eqs. (1-2) will be written as:

$$
\begin{aligned}
N(x) & =N=\text { const }, Q(x)=Q_{-l}-\frac{N}{R} \frac{x+l}{1 ! l}-P H(x) \\
M(x) & =M_{-l}+Q_{-l} \frac{x+l}{1 ! l}-\frac{N}{R} \frac{(x+l)^{2}}{2 ! l^{2}}-P \frac{x}{1 ! l} H(x) \\
\vartheta(x) & =\vartheta_{-l}+\frac{1}{E J}\left(M_{-l} \frac{x+l}{1 ! l}+Q_{-l} \frac{(x+l)^{2}}{2 ! l^{2}}-\frac{N}{R} \frac{(x+l)^{3}}{3 ! l^{3}}-P \frac{x^{2}}{2 ! l^{2}} H(x)\right) \\
w(x) & =w_{-l}+\vartheta_{-l} \frac{x+l}{1 ! l}+\frac{1}{E J}\left(M_{-l} \frac{(x+l)^{2}}{2 ! l^{2}}+Q_{-l} \frac{(x+l)^{3}}{3 ! l^{3}}-\frac{N}{R} \frac{(x+l)^{4}}{4 ! l^{4}}-P \frac{x^{3}}{3 ! l^{3}} H(x)\right) \\
u(x) & =u_{-l}-\frac{w_{-l}}{R} \frac{x+l}{1 ! l}-\frac{\vartheta_{-l}}{R} \frac{(x+l)^{2}}{2 ! l^{2}}- \\
& -\frac{1}{E J R}\left(M_{-l} \frac{(x+l)^{3}}{3 ! l^{3}}+Q_{-l} \frac{(x+l)^{4}}{4 ! l^{4}}-\frac{N}{R} \frac{(x+l)^{5}}{5 ! l^{5}}-P \frac{x^{4}}{4 ! l^{4}} H(x)\right)
\end{aligned}
$$

where $H(x)$ is Heaviside function.

The uknown constants $N, Q_{-l}, M_{-l}, u_{-l}, w_{-l}, \vartheta_{-l} \vartheta_{-l}$ in expressions (9), as well as a linear connection between $P$ and $\Delta$ for each of the six schemes we find from a specific group of boundary conditions (3-8). Neglecting the values of the second order of smallness against one, we will put down the final results. Thus, for the scheme 1 solution of the boundary problem (1-3) is:

$$
\begin{aligned}
& N(x)=-P \frac{f}{l}, Q(x)=P\left(\frac{1}{2}-H(x)\right), M(x)=P l\left(\frac{1}{2} \frac{x+l}{1 ! l}-\frac{x}{1 ! l} H(x)\right), \\
& \vartheta(x)=\frac{P l^{2}}{E J}\left(-\frac{1}{4}+\frac{1}{2} \frac{(x+l)^{2}}{2 ! l^{2}}-\frac{x^{2}}{2 ! l^{2}} H(x)\right), \\
& w(x)=\frac{P l^{3}}{E J}\left(-\frac{1}{4} \frac{x+l}{1 ! l}+\frac{1}{2} \frac{(x+l)^{3}}{3 ! l^{3}}-\frac{x^{3}}{3 ! l^{3}} H(x)\right), \\
& u(x)=\frac{2 P l^{2} f}{E J}\left(\frac{1}{4} \frac{(x+l)^{2}}{2 ! l^{2}}-\frac{1}{2} \frac{(x+l)^{4}}{4 ! l^{4}}+\frac{x^{4}}{4 ! l^{4}} H(x)\right)
\end{aligned}
$$


$P=\frac{6 E J}{l^{3}} \Delta$.

For the scheme 2 solution of the boundary problem (1), (2), (4) is: writed as::

$N(x)=-\frac{25}{64} P \frac{f}{l}, Q(x)=P\left(-\frac{9}{32}+\frac{25}{32} \frac{x+l}{1 ! l}-H(x)\right)$,

$M(x)=P l\left(-\frac{9}{32} \frac{x+l}{1 ! l}+\frac{25}{32} \frac{(x+l)^{2}}{2 ! l^{2}}-\frac{x}{1 ! l} H(x)\right)$,

$\vartheta(x)=\frac{P l^{2}}{E J}\left(\frac{1}{96}-\frac{9}{32} \frac{(x+l)^{2}}{2 ! l^{2}}+\frac{25}{32} \frac{(x+l)^{3}}{3 ! l^{3}}-\frac{x^{2}}{2 ! l^{2}} H(x)\right)$,

$w(x)=\frac{P l^{3}}{E J}\left(\frac{1}{96} \frac{x+l}{1 ! l}-\frac{9}{32} \frac{(x+l)^{3}}{3 ! l^{3}}+\frac{25}{32} \frac{(x+l)^{4}}{4 ! l^{4}}-\frac{x^{3}}{3 ! l^{3}} H(x)\right)$,

$u(x)=\frac{2 P l^{2} f}{E J}\left(-\frac{1}{96} \frac{(x+l)^{2}}{2 ! l^{2}}+\frac{9}{32} \frac{(x+l)^{4}}{4 ! l^{4}}-\frac{25}{32} \frac{(x+l)^{5}}{5 ! l^{5}}+\frac{x^{4}}{4 ! l^{4}} H(x)\right)$;

$P=256 \frac{E J}{l^{3}} \Delta$.

For the scheme 3 solution of the boundary problem (1), (2), (5) is:

$N(x)=-\frac{5}{8} P \frac{f}{l}, Q(x)=P\left(\frac{11}{16}-H(x)\right), M(x)=P l\left(-\frac{3}{8}+\frac{11}{16} \frac{x+l}{1 ! l}-\frac{x}{1 ! l} H(x)\right)$,

$\vartheta(x)=\frac{P l^{2}}{E J}\left(-\frac{3}{8} \frac{x+l}{1 ! l}+\frac{11}{16} \frac{(x+l)^{2}}{2 ! l^{2}}-\frac{x^{2}}{2 ! l^{2}} H(x)\right)$,

$w(x)=\frac{P l^{3}}{E J}\left(-\frac{3}{8} \frac{(x+l)^{2}}{2 ! l^{2}}+\frac{11}{16} \frac{(x+l)^{3}}{3 ! l^{3}}-\frac{x^{3}}{3 ! l^{3}} H(x)\right)$,

$u(x)=\frac{2 P l^{2} f}{E J}\left(\frac{3}{8} \frac{(x+l)^{3}}{3 ! l^{3}}-\frac{11}{16} \frac{(x+l)^{4}}{4 ! l^{4}}+\frac{x^{4}}{4 ! l^{4}} H(x)\right) ;$

$P=\frac{96}{7} \frac{E J}{l^{3}} \Delta$

For the scheme 4 solution of the boundary problem (1), (2), (6) is written as:

$$
\begin{aligned}
& N(x)=-\frac{5}{12} P \frac{l}{f}, Q(x)=P\left(-\frac{17}{48}+\frac{5}{6} \frac{x+l}{1 ! l}-H(x)\right), \\
& M(x)=P l\left(\frac{1}{24}-\frac{17}{48} \frac{x+l}{1 ! l}+\frac{5}{6} \frac{(x+l)^{2}}{2 ! l^{2}}-\frac{x}{1 ! l} H(x)\right), \\
& \vartheta(x)=\frac{P l^{2}}{E J}\left(\frac{1}{24} \frac{x+l}{1 ! l}-\frac{17}{48} \frac{(x+l)^{2}}{2 ! l^{2}}-\frac{5}{6} \frac{(x+l)^{3}}{3 ! l^{3}} \frac{x^{2}}{2 ! l^{2}} H(x)\right),
\end{aligned}
$$




$$
\begin{aligned}
& w(x)=\frac{P l^{3}}{E J}\left(\frac{1}{24} \frac{(x+l)^{2}}{2 ! l^{2}}-\frac{17}{48} \frac{(x+l)^{3}}{3 ! l^{3}}+\frac{5}{6} \frac{(x+l)^{4}}{4 ! l^{4}}-\frac{x^{3}}{3 ! l^{3}} H(x)\right) \\
& u(x)=\frac{2 P l^{2} f}{E J}\left(-\frac{1}{24} \frac{(x+l)^{3}}{3 ! l^{3}}+\frac{17}{48} \frac{(x+l)^{4}}{4 ! l^{4}}+\frac{5}{6} \frac{(x+l)^{5}}{5 ! l^{5}}-\frac{x^{4}}{4 ! l^{4}} H(x)\right) \\
& P=288 \frac{E J}{l^{3}} \Delta .
\end{aligned}
$$

For the scheme 5 solution of the boundary problem (1), (2), (7) will be:

$$
\begin{aligned}
& N(x)=-P \frac{f}{l}, Q(x)=P\left(\frac{1}{2}-H(x)\right), M(x)=P l\left(-\frac{1}{4}+\frac{1}{2} \frac{x+l}{1 ! l}-\frac{x}{1 ! l} H(x)\right), \\
& \vartheta(x)=\frac{P l^{2}}{E J}\left(-\frac{1}{4} \frac{x+l}{1 ! l}+\frac{1}{2} \frac{(x+l)^{2}}{2 ! l^{2}}-\frac{x^{2}}{2 ! l^{2}} H(x)\right), \\
& w(x)=\frac{P l^{3}}{E J}\left(-\frac{1}{4} \frac{(x+l)^{2}}{2 ! l^{2}}+\frac{1}{2} \frac{(x+l)^{3}}{3 ! l^{3}}-\frac{x^{3}}{3 ! l^{3}} H(x)\right), \\
& u(x)=\frac{2 P l^{2} f}{E J}\left(\frac{1}{4} \frac{(x+l)^{3}}{3 ! l^{3}}-\frac{1}{2} \frac{(x+l)^{4}}{4 ! l^{4}}+\frac{x^{4}}{4 ! l^{4}} H(x)\right) \\
& P=24 \frac{E J}{l^{3}} \Delta .
\end{aligned}
$$

Finally, for the scheme 6 solution of the boundary problem (1), (2), (8) is:

$$
\begin{aligned}
& N(x)=-\frac{15}{32} P \frac{f}{l}, Q(x)=P\left(-\frac{7}{16}+\frac{15}{16} \frac{x+l}{1 ! l}-H(x)\right), \\
& M(x)=P l\left(\frac{1}{16}-\frac{7}{16} \frac{x+l}{1 ! l}+\frac{15}{16} \frac{(x+l)^{2}}{2 ! l^{2}}-\frac{x}{1 ! l} H(x)\right), \\
& \vartheta(x)=\frac{P l^{2}}{E J}\left(\frac{1}{16} \frac{x+l}{1 ! l}-\frac{7}{16} \frac{(x+l)^{2}}{2 ! l^{2}}+\frac{15}{16} \frac{(x+l)^{3}}{3 ! l^{3}}-\frac{x^{2}}{2 ! l^{2}} H(x)\right), \\
& w(x)=\frac{P l^{3}}{E J}\left(\frac{1}{16} \frac{(x+l)^{2}}{2 ! l^{2}}-\frac{7}{16} \frac{(x+l)^{3}}{3 ! l^{3}}+\frac{15}{16} \frac{(x+l)^{4}}{4 ! l^{4}}-\frac{x^{3}}{3 ! l^{3}} H(x)\right), \\
& u(x)=\frac{2 P l^{2} f}{E J}\left(-\frac{1}{16} \frac{(x+l)^{3}}{3 ! l^{3}}+\frac{7}{16} \frac{(x+l)^{4}}{4 ! l^{4}}-\frac{15}{16} \frac{(x+l)^{5}}{5 ! l^{5}}+\frac{x^{4}}{4 ! l^{4}} H(x)\right) \\
& P=384 \frac{E J}{l^{3}} \Delta .
\end{aligned}
$$

The bearing capacity of rods is evaluated according to the maximum normal stresses theory (Gere \& Goodno, 2012): 


$$
\sigma_{e q}=\max _{x}|\sigma(x)|=\max _{x}\left|\frac{N(x)}{F} \pm \frac{M(x) h}{2 J}\right| \leq[\sigma],
$$

where $[\sigma]$ is admissible stress for rod material, $F=b h$ and $J=b h^{3} / 12$ are area and inertia moment of the cross-section.

Results of calculations performed for all schemes by formulas (10-22) when $f / l=0,1$, $h / l=0,024$, are provided in Figs. 5-10.
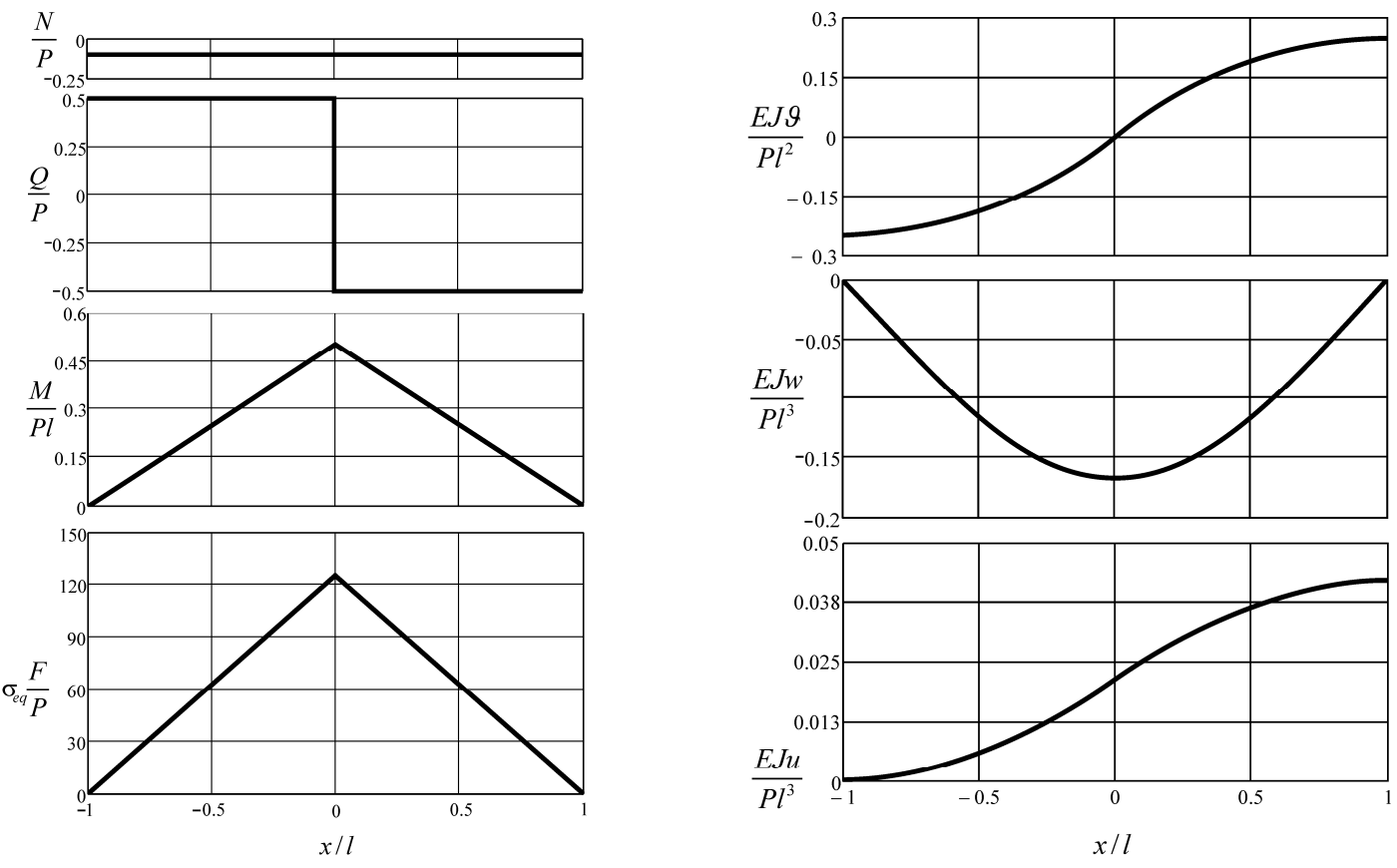

Fig. 5. Distribution of tangential and transverse forces, bending moment and equivalent stress, angle of rotation, normal and tangential displacements in the elastic link of a hinged movable centralizer (scheme 1).
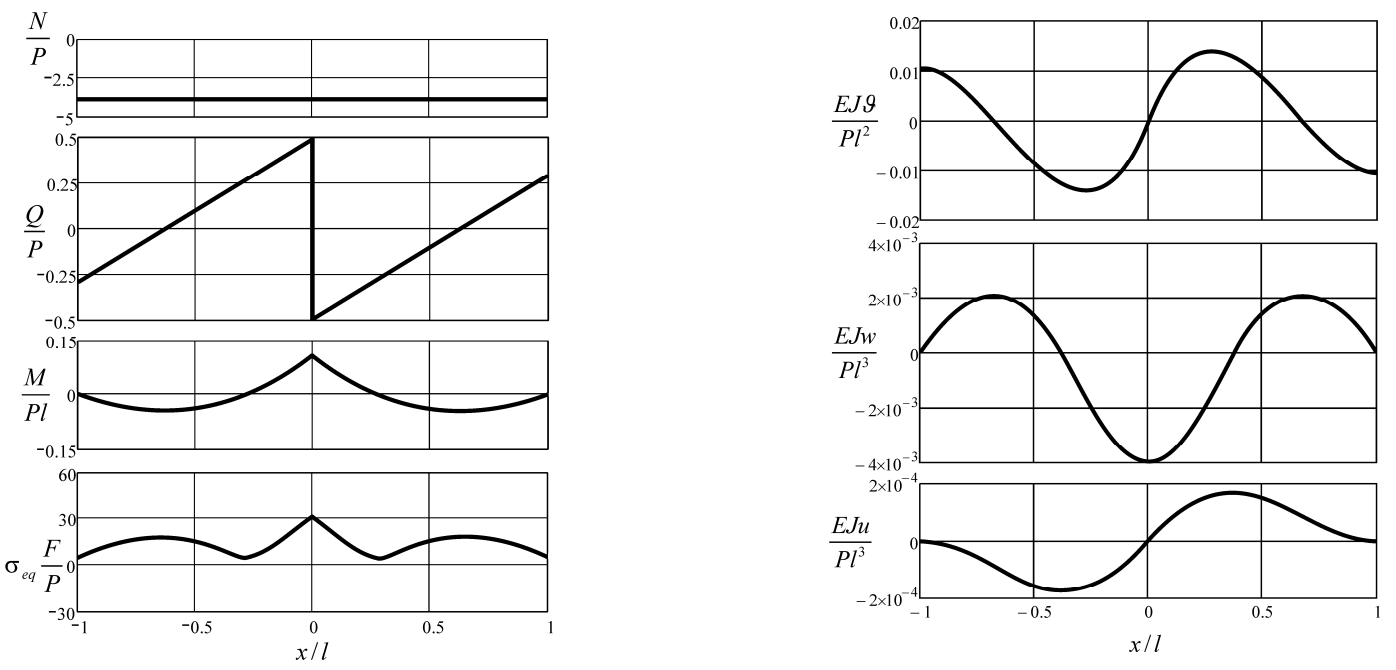

Fig. 6. Distribution of tangential and transverse forces, bending moment and equivalent stress, angle of rotation, normal and tangential displacements in the elastic link of a hinged fixed centralizer (scheme 2) 

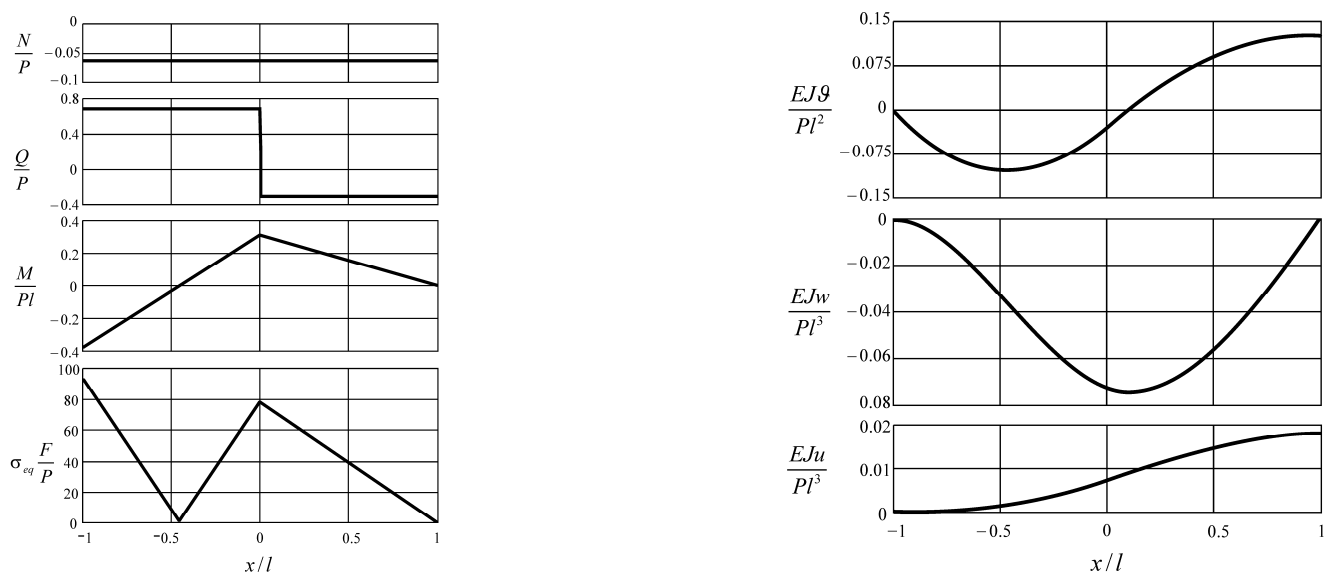

Fig. 7. Distribution of tangential and transverse forces, bending moment and equivalent stress, angle of rotation, normal and tangential displacements in the elastic link of a cast movable centralizer (scheme 3 ).
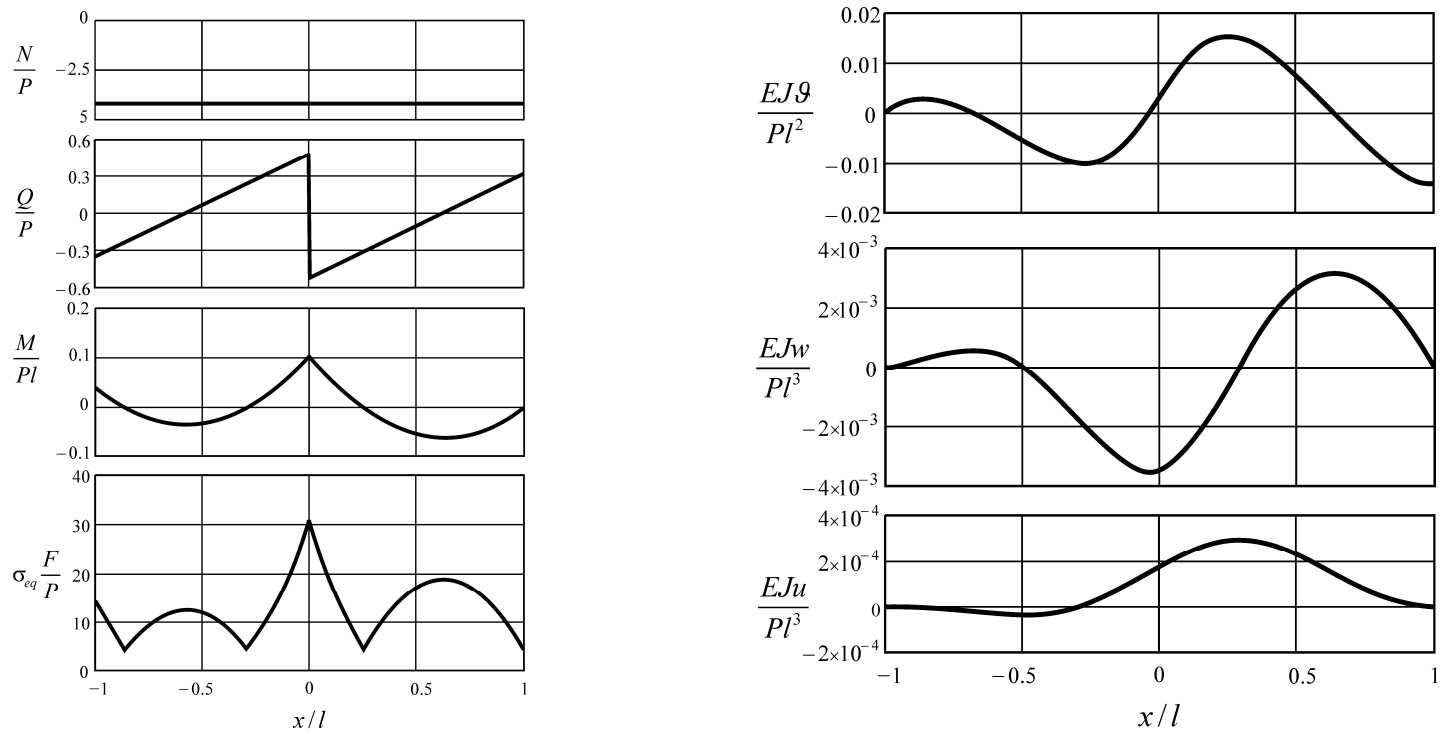

Fig. 8. Distribution of tangential and transverse forces, bending moment and equivalent stress, angle of rotation, normal and tangential displacements in the elastic link of a cast fixed centralizer (scheme 4 ).
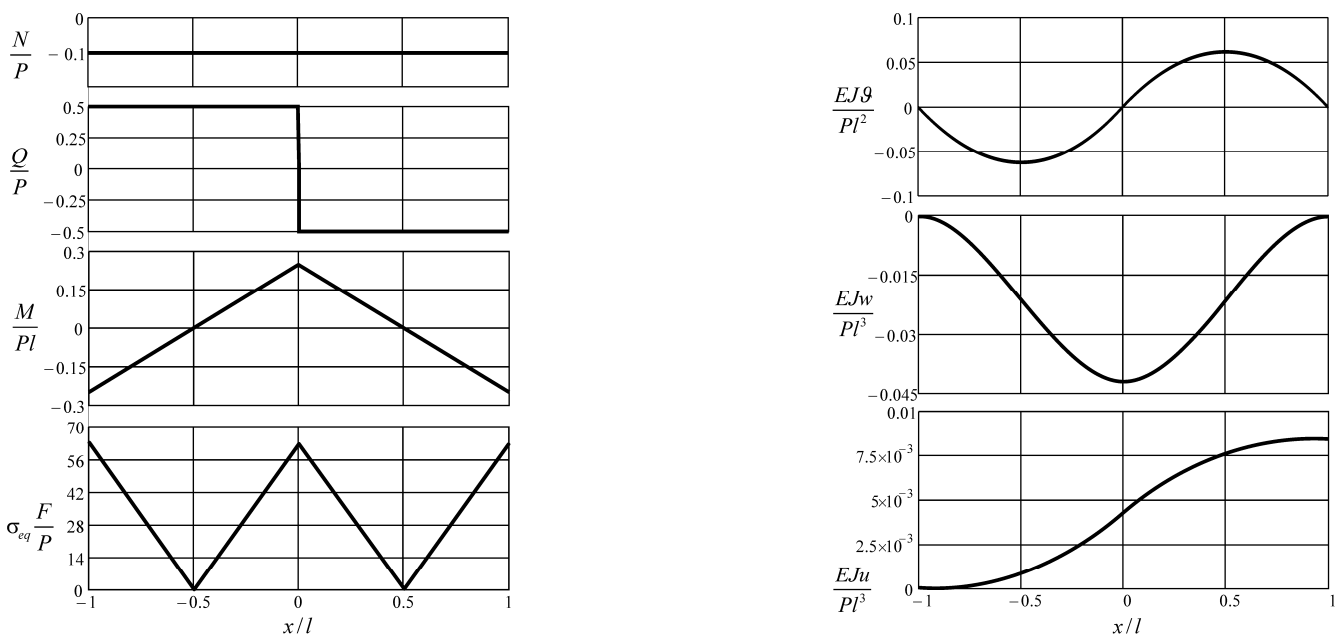

Fig. 9. Distribution of tangential and transverse forces, bending moment and equivalent stress, angle of rotation, normal and tangential displacements in the elastic link of a welded movable centralizer (scheme 5). 

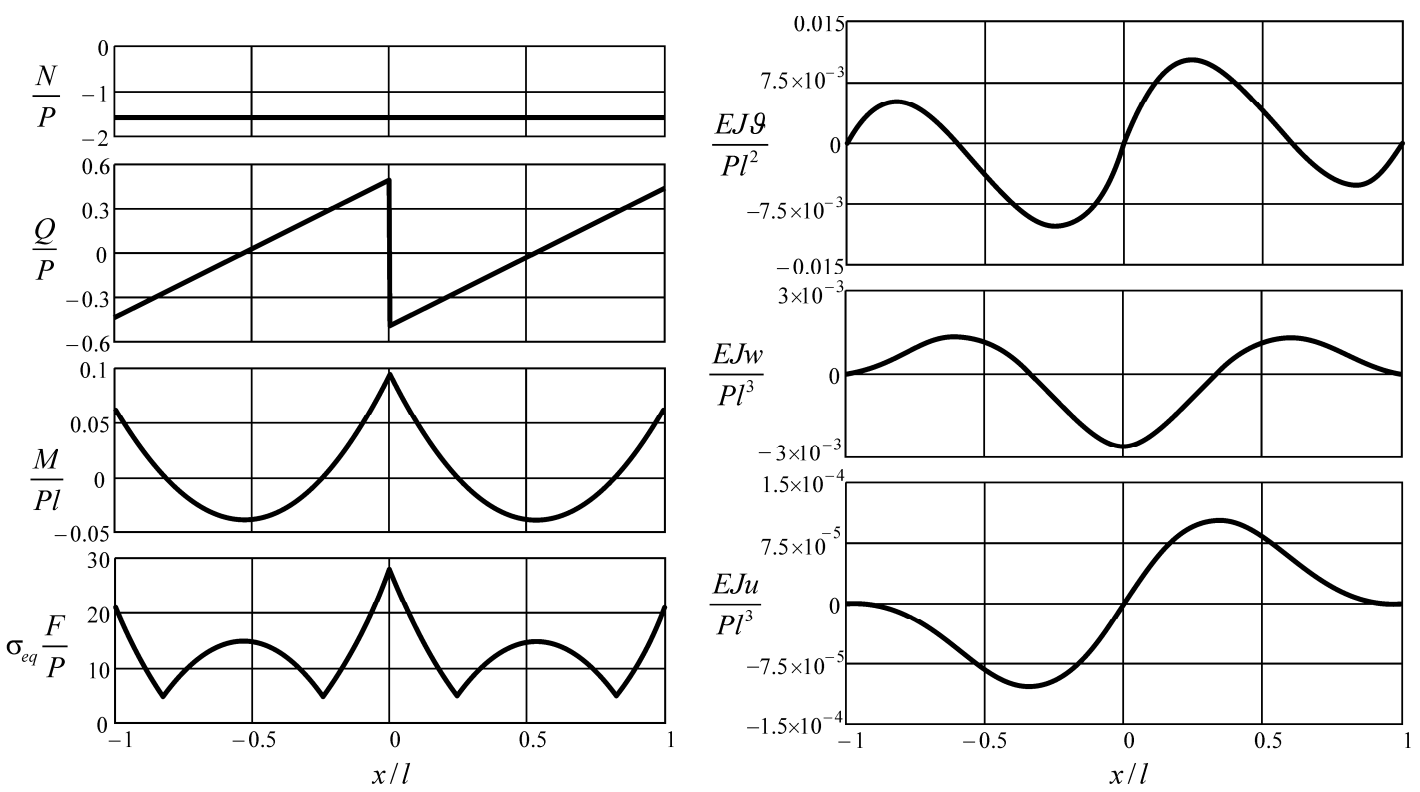

Fig. 10. Distribution of tangential and transverse forces, bending moment and equivalent stress, angle of rotation, normal and tangential displacements in the elastic link of a welded fixed centralizer (scheme 6 ).

\subsection{Comparison of Mechanical Properties of Centralizers}

Let's compare we the contact rigidities of structures. Formulas (11), (13), (15), (17), (19) and (21) can be written as: $P=C_{i} \Delta$,

where $C_{1}=6 \frac{E J}{l^{3}}, C_{2}=256 \frac{E J}{l^{3}}, C_{3}=\frac{96}{7} \frac{E J}{l^{3}}, C_{4}=288 \frac{E J}{l^{3}}, C_{5}=24 \frac{E J}{l^{3}}, C_{6}=384 \frac{E J}{l^{3}}-$ coefficients of rigidity, and the value of the index $i$ corresponds to the scheme number in Figs. 2-4.

Thus, the prohibition of the mutual movement of supports along the pipe in the hinged centralizer increases its rigidity $C_{2} / C_{1}=256: 6 \approx 42,67$ times. The real rigidity of the hinged centralizer, taking into account the joint operation of its links $C_{c}$ lies in the range $C_{1} \leq C_{c} \leq C_{2}$. On the other hand, the prohibition of the mutual movement of supports along the pipe in the cast centralizer increases its rigidity $C_{4} / C_{3}=288:(96 / 7)=21$ times. The real rigidity of a cast centralizer with unlimited longitudinal displacement $C_{c}=C_{3}$, since its links do not interact, and in this case there is no need for spatial calculation. Finally, limiting the axial displacement of rings in a welded centralizer increases its rigidity $C_{6} / C_{5}=384: 24=16$ times. In this case $C_{5} \leq C_{c} \leq C_{6}$. By comparing the rigidity of the rods according to schemes 1,3 and 5 , we determine that the cast and welded centralizers with free axial displacement are more rigid than similar hinged structures: $C_{3} / C_{1}=(96 / 7): 6=16 / 7 \approx 2,29$, $C_{5} / C_{1}=24: 6=4$, and the rigidity of the welded centralizer exceeds the rigidity of the cast one: $C_{5} / C_{3}=24:(96 / 7)=7 / 4=1,75$. A similar comparison for the fixed rods in the longitudinal direction will be: $C_{4} / C_{2}=288: 256=9 / 8 \approx 1,13, \quad C_{6} / C_{2}=384: 256=3 / 2=1,5$, $C_{6} / C_{4}=384: 288==4 / 3 \approx 1,33$. Let's pass over to the assessment of the strength of the centralizer links. Analysis of the results (10), (12), (14), (16), (18), (20) and (22) for equivalent stresses, carried out when $f / l=0,1, h / l=0,024$, shows, that neighborhood of point $x=0$ in the middle of the rod is dangerous for schemes $1,2,4,5,6$ and only for scheme 3 -left fixed rod end $x=-l$. With the precision taken here, we obtain analytical estimates for the admissible forces of pressing: $P \leq P_{i}$, where 


$$
\begin{aligned}
& P_{1}=\frac{1}{3} \frac{[\sigma] b h^{2}}{l}, P_{2}=\frac{64}{42+25 \frac{h}{f}} \frac{[\sigma] b h^{2}}{l}, P_{3}=\frac{4}{9} \frac{[\sigma] b h^{2}}{l}, \\
& P_{4}=\frac{24}{15+10 \frac{h}{f}} \frac{[\sigma] b h^{2}}{l}, P_{5}=\frac{2}{3} \frac{[\sigma] b h^{2}}{l}, P_{6}=\frac{32}{18+15 \frac{h}{f}} \frac{[\sigma] b h^{2}}{l}
\end{aligned}
$$

are admissible loads, $i$ is scheme number.

Let us compare the admissible loads for axially movable and axially fixed structures:

$$
\frac{P_{2}}{P_{1}}=\frac{64}{42+25 \frac{h}{f}}: \frac{1}{3} \approx 4, \frac{P_{4}}{P_{3}}=\frac{24}{15+10 \frac{h}{f}}: \frac{4}{9} \approx 3,1, \frac{P_{6}}{P_{5}}=\frac{32}{18+15 \frac{h}{f}}: \frac{2}{3} \approx 2,22 .
$$

When comparing hinged, cast and welded centralizers by the admissible load we will get the following results:

for movable schemes

$$
\frac{P_{3}}{P_{1}}=\frac{4}{9}: \frac{1}{3} \approx 1,33, \frac{P_{5}}{P_{1}}=\frac{2}{3}: \frac{1}{3}=2, \frac{P_{5}}{P_{3}}=\frac{2}{3}: \frac{4}{9}=1,5,
$$

and when there are no axial displacements of supports

$$
\frac{P_{4}}{P_{2}}=\frac{24}{15+10 \frac{h}{f}}: \frac{64}{42+25 \frac{h}{f}} \approx 1,03, \frac{P_{6}}{P_{2}}=\frac{32}{18+15 \frac{h}{f}}: \frac{64}{42+25 \frac{h}{f}} \approx 1,15, \frac{P_{6}}{P_{4}}=\frac{32}{18+15 \frac{h}{f}}: \frac{24}{15+10 \frac{h}{f}} \approx 1,07 .
$$

\section{Conclusions}

Models of cyclically symmetric rod systems for analytical estimations of the stress state, strength and rigidity of casing pipe centralizers are developed. The contact problems for six variants of designs of centralizers are formulated and solved. We have determined analytical relationships between the clamping force and the mutual convergence of the column and the well wall, which characterize the radial rigidity of the centralizer, as well as expressions for the maximum stresses that serve to assess its strength. The way of fastening the rod along the axis of the pipe significantly influences these characteristics; in particular, the presence or absence of freedom of mutual movement of the ends of the rod in the axial direction is decisive. Simple engineering formulas that are two-way estimates of the rigidity and strength of the real designs of the hinged, cast and welded centering devices are obtained for these two cases. In order to clarify the obtained results, it is necessary to construct spatial models for calculating centralizers as a connected system of rod links.

\section{References}

Aleksandrov, M. M. (1982). Interaction of the pipe columns with the walls of the well. Moscow: Nedra.

Bedzir, A. A., Shatskii, I. P., \& Shopa, V. M. (1995). Nonideal contact in a composite shell structure with a deformable filler. International Applied Mechanics, 31(5), 351-354.

Casing Accessories. (2016). Cement integrity starts here. Well construction. Weatherford.

Fryz, I. M. (2003). Centralizer for casing. Kiev: Interpres Ltd.

Gere, J., \& Goodno, B. (2012). Mechanics of materials. Stamford: Cengage Learning.

Larin, P. A., Gutorov, Yu. A., \& Yakunina, S. N. (2013). Problems of qualitative cementing of wells with intricate profile. Oktiabrskyi: RKNTC.

Levchuk, K. G. (2018). Engineering tools and technologies of freeing of the stuck metal drilling string. Metallofizika i Noveishie Tekhnologii, 40(1), 45-137.

Liu, G., \& Weber, L. D. (2012). Centralizer Selection Placement Optimization. SPE Deepwater Drilling and 
Completions Conference. Society of Petroleum Engineers. Galveston, Texas, USA.

Jawed, M. K., Novelia A., \& O'Reilly, O. M. (2018). Kirchhoff's theory of an elastic rod. In: A Primer on the Kinematics of Discrete Elastic Rods. SpringerBriefs in Applied Sciences and Technology. Springer, Cham.

Kozhevnikov, E. V., Nikolaev, N. I., Rozentsvet, A. V., \& Lyrchikov, A. A. (2015). Entering equipment for casing columns in sidetrack cementing. Bulletin of PNRPU. Geology. Oil \& Gas Engineering \& Mining, 16, $54-60$.

Kotskulych, Ya. S., Seniushkovych, M. V., Martsynkiv, O. B., \& Vytvytskyi, I. I. (2016). Casing string centering in directional wells. Naukovyi Visnyk Natsionalnoho Hirnychoho Universytetu, 2, 23-30.

Kryzhanivskyi, Ye. I., Paliichuk, I. I., \& Malyk, H. D. (2018). Large deformations of the casing string under its own weight in the curvilinear well. Naukovyi Visnyk Natsionalnoho Hirnychoho Universytetu, 6, 72-79.

Money Tuli, Balvinder Singh, \& Dr Rahul Malhotra. (2018). Design and analysis of centralizer used in wellbore. International Journal of Academic Research and Development, 13(2), 1448-1453.

Mossakowskii, V. I., Hudramovich, V. S., \& Makeev, E. M. (1978). Contact problems in the theory of shells and rods. Moscow: Mashinostroienie.

Neto, A. G., Pimenta, P. M., \& Wriggers, P. (2014). Contact between rolling beams and flat surfaces. International Journal for Numerical Methods in Engineering, 97(9), 683-706.

Popadyuk, I. Yo., Shats'kyi I. P., Shopa V. M., \& Velychkovych A. S. (2016). Frictional interaction of a cylindrical shell with deformable filler under nonmonotonic loading. Journal of Mathematical Sciences, 215(2), 243-253.

Rekach, V. G. (2010). Guide to the solution of tasks of the applied theory of elasticity. Moscow: Librokom.

Renpu, W. (2011). Advanced Well Completion Engineering. 3rd edition. Elsevier Inc.

Shatskyi, I. P., Biletskyi, Ya. S., \& Vytvytskyi, I. I. (2014). Two-side estimates of rigidity and strength of casing centralizer. Odes 'kyi politekhnichnyi universytet. Pratsi, 1, 68-73.

Shats'kyi, I. P., Lyskanych, O. M., \& Kornuta, V. A. (2016). Combined deformation conditions for fatigue damage indicator and well-drilling tool joint. Strength of Materials, 48(3), 469-472.

Shats'kyi, I. P. (2005). Closure of a longitudinal crack in a shallow cylindrical shell in bending. Materials Science, 41(2), 186-191.

Shats'kyi, I. P., \& Makoviichuk, M. V. (2005). Contact interaction of crack lips in shallow shells in bending with tension. Materials Science, 41(4), 486-494.

Shats'kyi, I. P., \& Makoviichuk, M. V. (2009). Analysis of the limiting state of cylindrical shells with cracks with regard for the contact of crack lips. Strength of Materials, 41(5), 560-565.

Shatskii, I. P., \& Makoviichuk, N. V. (2011). Effect of closure of collinear cracks on the stress-strain state and the limiting equilibrium of bent shallow shells. Journal of Applied Mechanics and Technical Physics, 52(3), 464470.

Vytvytskyi, I. I., Seniushkovych, M. V., \& Shatskyi, I. P. (2017). Calculation of distance between elastic-rigid centralizers of casing. Naukovyi Visnyk Natsionalnoho Hirnychoho Universytetu, 5, 28-35.

Vlasiy, O., Mazurenko, V., Ropyak, L., \& Rogal, O. (2017). Improving the aluminum drill pipes stability by optimizing the shape of protector thickening. Eastern-European Journal of Enterprise Technologies, 1(7), 2531.

Tikhonov, V. S., Bukashkina, O. S., \& Gandikota, R. (2014). Numerical simulation of casing centralization. In: 11th World Congress on Computational Mechanics (WCCM XI) and 5th European Conference on Computational Mechanics (ECCM V) and 6th European Conference on Computational Fluid Dynamics (ECFD VI). Barcelona, Spain.

Velichkovich, A. S., \& Dalyak, T. M. (2015). Assessment of stressed state and performance characteristics of jacketed spring with a cut for drill shock absorber. Chemical and Petroleum Engineering, 51(3), 188-193.

Velichkovich, A., Dalyak, T., \& Petryk, I. (2018). Slotted shell resilient elements for drilling shock absorbers. Oil \& Gas Science and Technology - Rev. IFP Energies nouvelles, 73 (34), 1-8.

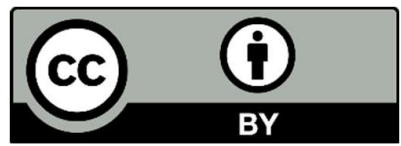

(C) 2019 by the authors; licensee Growing Science, Canada. This is an open access article distributed under the terms and conditions of the Creative Commons Attribution (CC-BY) license (http://creativecommons.org/licenses/by/4.0/). 